\title{
Is G-CSF Dangerous in COVID-19: Why Not Use GM-CSF?
}

\author{
Hillard M. Lazarus ${ }^{a}$ Robert Peter Gale ${ }^{b}$ \\ ${ }^{a}$ Department of Medicine, Division of Hematology and Oncology, Case Western Reserve University, Cleveland, $\mathrm{OH}, \mathrm{USA}$; \\ ${ }^{b}$ Centre for Haematology, Department of Immunology and Inflammation, Imperial College London, London, UK
}

We recently published an article in Acta Haematologica (DOI: 10.1159/000510352) [1] wherein we compared safety and efficacy of G-CSF and GM-CSF therapy in persons with acute respiratory distress syndrome (ARDS) such as that associated with infection with severe acute respiratory syndrome-coronavirus 2 (SARS-CoV-2) and attendant coronavirus disease-2019 (COVID-19). We discussed differences between the drugs and suggested that in persons with lung infection and/or ARDS, GMCSF may be safer than G-CSF because of its pleiotropic effects. There are now 2 publications emphasizing the potential danger of giving G-CSF to persons with COVID19 -related ARDS [2,3]. Nawar and colleagues [2] reported 3 persons with ARDS in the setting of COVID-19 with rapid deterioration of lung function after receiving GCSF. They attribute this finding to an influx of granulocytes into the inflamed lung concluding: In the setting of COVID-19 illness, further rapid rise in neutrophilia with NLR (neutrophil lymphocyte ratio) $>5$ may portend respiratory deterioration to the point of mechanical ventilation within the next $72 \mathrm{~h}$, especially in those patients who are older than age 50 and have comorbid medical conditions. Taha and colleagues [3] report similar rapid dete- rioration in someone with COVID-19-related ARDS receiving G-CSF concluding: This rapid neutropenia recovery and the robust inflammatory response in COVID-19 raise concerns about G-CSF safety in patients with $\mathrm{CO}$ VID-19.

We ended our article noting: In persons with lung infection and/or ARDS, GM-CSF may be a safer drug than G-CSF. Whether this is so can only be definitively answered in a randomized comparative trial. Unfortunately, such a trial is unlikely to be done and we may have to rely on indirect evidence of safety and efficacy, perhaps to the chagrin of North et al. [4], who advocate enhancing enrollment to clinical trials, especially during the pandemic. The articles we cite on use of G-CSF in this setting are perhaps a warning and the kind of indirect evidence needed.

\section{Acknowledgement}

R.P.G. acknowledges support from the National Institute of Health Research (NIHR) Biomedical Research Centre funding scheme. karger@karger.com

www.karger.com/aha

(c) 2020 S. Karger AG, Basel

Karger!
Hillard M. Lazarus

Department of Medicine, Case Western Reserve University

10900 Euclid Avenue

Cleveland, OH 44106 (USA)

Hillard.Lazarus@ case.edu 


\section{Conflict of Interest Statement}

H.M.L. has been a consultant for Partner Therapeutics, Jazz Pharmaceuticals, Seattle Genetics, AstraZeneca, Celgene/ Bristol-Myers Squibb, and Actinium Pharmaceuticals. R.P.G. is consultant to: BeiGene Ltd., Kite Pharma Inc., Fusion Phar- ma LLC, LaJolla NanoMedical Inc., Mingsight Parmaceuticals Inc., and CStone Pharmaceuticals. Medical Director: FFF Enterprises Inc. Partner: Neopharm Ltd, AZACA Inc. Board of Directors: RakFond Foundation for Cancer Research Support. Scientific Advisory Board: Antegene Biotech LLC, StemRad Ltd.

\section{References}

Is G-CSF Dangerous in COVID-19: Why Not Use GM-CSF?
1 Lazarus HM, Gale RP. G-CSF and GM-CSF Are Different. Which One Is Better for COVID-19? Acta Haematol. 2020 Aug;13:1-4.

2 Nawar T, Morjaria S, Kaltsas A, Patel D, Perez-Johnston R, Daniyan AF, et al. Granulocyte-colony stimulating factor in COVID-19: is it stimulating more than just the bone marrow? Am J Hematol. 2020 Aug;95(8):E210-3.
3 Taha M, Sharma A, Soubani A. Clinical deterioration during neutropenia recovery after G-CSF therapy in patient with COVID-19. Respir Med Case Rep. 2020;31:101231.

4 North CM, Dougan ML, Sacks CA. Improving clinical trial enrollment - In the Covid-19 era and beyond. N Engl J Med. 2020 Oct; 383(15):1406-8 